\title{
Expression profile of CENH3 paralogs in different tissues of the rye-wheat amphiploids and their parents
}

\author{
Gatzkaya S.S.*, Evtushenko E.V. \\ Institute of Molecular and Cellular Biology SB RAS, Novosibirsk, Russia \\ *e-mail: jait@mail.ru
}

Key words: Centromeric histone CENH3, gene expression, rye, secalotriticum, remote hybridization

Motivation and Aim: It was established that centromeric histone H3 (CENH3 in plants) plays a major role in the formation and proper functioning of centromeres. The created allopolyploid hybrids secalotritikum (rye $\mathrm{x}$ wheat) are a new interesting model for studying the effect of remote hybridization on the genome regions that are responsible for the correct division and distribution of chromosomes in hybrid cells. The aim of this work was to compare the expression levels of two forms of centromeric histone H3 ( $\alpha \mathrm{CENH} 3$ and $\beta C E N H 3$ ) in stable rye-wheat hybrids secalotriticum and their parental species.

Methods and Algorithms: cDNA samples were isolated from vegetative and generative tissues of two varieties of secalotriticum (Secalotriticum Verasen $\times$ Mikhas and Secalotriticum Verasen $\times$ Dubrava) and parental species (tetraploid rye $S$. cereale v. Verasen and hexaploid hybrids Triticale v. Mikhas and Triticale v. Dubrava). Relative expression levels were determined by RT-PCR with SYBR Green I. Reference gene Ta2776 was used to normalize expression levels of the CENH3 genes. Calculations were performed using the LC480 software (Roche) by 'Relative Quantification analysis with high confidence 2nd Derivative max'.

Results: The main forms of the centromeric histone $\mathrm{H} 3$ gene, $\alpha C E N H 3$ and $\beta C E N H 3$, are expressed at all stages of individual plant development in both hybrids and in their parents. The transcriptional activity profiles of $\alpha C E N H 3$ and $\beta C E N H 3$ are almost identical in secalotriticum hybrids and the parental species. In all the studied tissues the expression level of $\alpha C E N H 3$ usually an order of magnitude higher than the expression level of $\beta C E N H 3$. There is a significant decrease in the expression levels of both $C E N H 3$ forms in vegetative tissues after germination, namely in the leaf tissue expression levels 8 times lower, on average, compared to the tissues of radicle and coleoptile. Expression levels in the generative tissue increase many times compared to vegetative tissue, and the expression of both forms is 2-3 times higher in the pistil tissue than in the anther tissue.

Conclusion: The activity of the CENH3 genes remains at the same level in genomes of the stable secalotriticum hybrids compared to genomes of the parental species, that is, the hybrid cytoplasm does not affect this feature. These results reflect the ability of centromeres to adapt to a new genetic environment. Acknowledgements: Supported by the Russian Foundation for Basic Research (grant 20-04-00699 A). 